\title{
miRNA-183 Suppresses Apoptosis and Promotes Proliferation in Esophageal Cancer by Targeting PDCD4
}

\author{
Miao Yang ${ }^{1}$, Ran Liu', Xiajun Li', Juan Liao',Yuepu Pu', Enchun Pan', Lihong Yin ${ }^{1, *}$, and Yi Wang ${ }^{2, *}$
}

In our previous study, miRNA-183, a miRNA in the miR-96182-183 cluster, was significantly over-expressed in esophageal squamous cell carcinoma (ESCC). In the present study, we explored the oncogenic roles of miR-183 in ESCC by gain and loss of function analysis in an esophageal cancer cell line (EC9706). Genome-wide mRNA microarray was applied to determine the genes that were regulated directly or indirectly by miR-183. 3'UTR luciferase reporter assay, RT-PCR, and Western blot were conducted to verify the target gene of miR-183. Cell culture results showed that miR-183 inhibited apoptosis $(p<0.05)$, enhanced cell proliferation $(p<0.05)$, and accelerated G1/S transition $(p<0.05)$. Moreover, the inhibitory effect of miR183 on apoptosis was rescued when miR-183 was suppressed via miR-183 inhibitor $(p<0.05)$. Western blot analysis showed that the expression of programmed cell death 4 (PDCD4), which was predicted as the target gene of miR183 by microarray profiling and bioinformatics predictions, decreased when miR-183 was over-expressed. The 3'UTR luciferase reporter assay confirmed that miR-183 directly regulated PDCD4 by binding to sequences in the 3'UTR of PDCD4. Pearson correlation analysis further confirmed the significant negative correlation between miR-183 and PDCD4 in both cell lines and in ESCC patients. Our data suggest that miR-183 might play an oncogenic role in ESCC by regulating PDCD4 expression.

\section{INTRODUCTION}

Esophageal cancer is one of the most common upper gastrointestinal tract cancers worldwide. Esophageal squamous cell carcinoma (ESCC) remains the most predominant type of

${ }^{1}$ Key Laboratory of Environmental Medicine Engineering, Ministry of Education, School of Public Health, Southeast University, Nanjing 210009, China, ${ }^{2}$ Huaian Center for Disease Control and Prevention, Huaian 223001, China

*Correspondence: Ihyin@seu.edu.cn (LY); wangyihuaian@163.com (YW)

Received 5 June, 2014; revised 20 September, 2014; accepted 22 September, 2014; published online 10 December, 2014

Keywords: apoptosis, esophageal cancer, miR-183, PDCD4, proliferation esophageal cancer in China, and the morbidity of esophageal adenocarcinoma is rapidly increasing in western countries (Guo et al., 2012; Hvid-Jensen et al., 2011; Lin et al., 2013). Data from 31 urban cancer registration areas in China in 2012 showed that ESCC is the $5^{\text {th }}$ leading cause of cancer morbidities and $4^{\text {th }}$ leading cause of cancer mortalities (He and Chen, 2012). Despite the development of improved therapeutic techniques, the overall survival for ESCC is still low (Dubecz et al., 2012). Low survival could be due to the diagnosis of patients at advanced stages, during which treatment options are very limited. The pathogenesis of ESCC must be elucidated to provide clues for early screening and prevention.

miRNAs are a class of non-coding RNAs that regulate gene expression by imperfect or perfect paring with target mRNAs, thereby inhibiting the translation and/or degrading the mRNAs (Calin and Croce, 2006). Results of previous studies support the concept that miRNAs can function as tumor suppressors or oncogenes by regulating downstream target genes (Fang et al., 2012; Patil et al., 2014). ESCC is a multifactorial disease caused by the complicated interaction between multiple genes and environmental features; miRNA is a major component of the epigenome and is correlated with changes in phenotypes of diseases (Borel and Antonarakis, 2008). Consequently, loss and gain of miRNAs may contribute to ESCC progression and the occurrence of malignant phenotypes. Several studies have shown that some specific miRNAs were aberrantly expressed in ESCC and participated in several biological processes by targeting different mRNAs (Hu et al., 2011; Yang et al., 2013). miR-183 was reported to be up-regulated in many cancers, including prostate cancer (Ueno et al., 2013), hepatocellular carcinoma (Li et al., 2010b), and bladder cancer (Han et al., 2011). Moreover, miR-183 was significantly up-regulated in ESCC, as we previously documented. However, miR-183 may also function as a tumor suppressor (Li et al., 2010a; Zhao et al., 2012; Zhu et al., 2012). As of this writing, information is lacking on the role of miR-183 in ESCC.

We explored the oncogenic role of miR-183 in ESCC and identified programmed cell death 4 (PDCD4) as a direct target gene of miR-183. Correlation analysis in a population study revealed that the expression of PDCD4 was associated with miR-183. Therefore, the findings of this study indicated the oncogenic role of miR-183 in ESCC tumorigenesis by targeting PDCD4, particularly in the apoptosis and proliferation processes.

elSSN: 0219-1032

(C) The Korean Society for Molecular and Cellular Biology. All rights reserved.

(c) This is an open-access article distributed under the terms of the Creative Commons Attribution-NonCommercial-ShareAlike 3.0 Unported License. To view a copy of this license, visit http://creativecommons.org/licenses/by-nc-sa/3.0/. 


\section{MATERIALS AND METHODS}

Specimen collection and ethics statement

A total of 81 patients were recruited from the First People's Hospital of Huaian between 2009 and 2010. All patients provided their consent and agreement. All patients were confirmed to have primary cancer by pathology and were not subjected to preoperative radiotherapy or chemotherapy. Tumor tissues and adjacent non-tumor tissues (located $\geq 5 \mathrm{~cm}$ from the edge of tumor tissues) were collected during the operation, treated with RNAlocker reagent (Tiandz, China), and stored at $-20^{\circ} \mathrm{C}$ until use. The present study was approved by the institutional review board (IRB of Southeast University Affiliated Zhongda Hospital) in Nanjing, China.

\section{Cell culture and transfection}

Two human ESCC cell lines and a human esophageal epithelial cell line were used in this study. EC109 and EC9706 were purchased from Shanghai Tiancheng Technology Co., Ltd. Het$1 \mathrm{~A}$ was purchased from Guangzhou Jennio Biotech Co., Ltd. EC109 and EC9706 were cultured in RPMl 1640 (HyClone, USA) containing $10 \%$ fetal bovine serum (HyClone, USA) and $1 \%$ penicillin/streptomycin (Sigma-Aldrich, USA) at $37^{\circ} \mathrm{C}$ with $5 \% \mathrm{CO}_{2}$, whereas Het-1A was cultured in Dulbecco's modified Eagle's medium (HyClone, USA). The miR-183 mimic and inhibitor were synthesized by RiboBio Corporation (China), and transfection was performed using Lipofectamine RNAiMAX (Life Technologies, USA) according to the instructions. Cells (2 $\times 10^{5}$ per well), miR-183 mimic with a final concentration of 30 $\mathrm{nM}$ or miR-183 inhibitor with a final concentration of $300 \mathrm{nM}$, and Lipofectamine RNAiMAX were seeded in a 6-well plate. Transfection efficiency was detected by RT-qPCR after $48 \mathrm{~h}$ of incubation.

\section{Genome-wide mRNA expression microarray}

Two pairs of EC9706 samples treated with miR-183 mimic or negative control (NC) were prepared for mRNA expression profiling analysis. Agilent Array platform $(4 \times 44 \mathrm{~K}$, Agilent Technologies) was used according to the manufacturer's standard protocols. Hierarchical clustering was performed to distinguish gene expression patterns among the samples. A 1.5-fold change cutoff was applied to filter the differentially expressed genes for data analysis. Genes with 1.5-fold higher expression than the control were considered as up-regulated, whereas genes with 1.5-fold lower expression were considered as downregulated. Pathway (http://www.genome.jp/kegg/) and gene ontology analyses (http://www.geneontology.org) were applied to determine the functions of these ectopically expressed genes in these biological pathways.

\section{Cell apoptosis}

Apoptosis rates were evaluated by flow cytometry (FACS Calibur, USA). After transfection for $48 \mathrm{~h}$, serum-containing medium was replaced by serum-free medium for $24 \mathrm{~h}$ to induce apoptosis. Cells were harvested and washed in cold PBS twice. Subsequently, $500 \mu \mathrm{l}$ buffer, $5 \mu \mathrm{l} \mathrm{FITC}$ annexin V, and $5 \mu \mathrm{l}$ propidium iodide $(\mathrm{PI})$ were added according to the established protocols (KeyGEN Biotech, China).

\section{EdU cell proliferation assay}

Proliferating EC9706 cells were determined using the cell-light 5-ethynyl-20-deoxyuridine (EdU) Apollo Imaging Kit (RiboBio, China) according to the manufacturer's protocol. EdU is a nucleoside analog of thymidine and is readily incorporated into
DNA during active DNA synthesis only by proliferating cells (Salic and Mitchison, 2008). Cells were incubated with $50 \mu \mathrm{M}$ EdU for 3 $\mathrm{h}$ after transfection. Subsequently, fixation, permeabilization, and EdU staining were performed. Nucleic acids in all cells were stained with Hoechst 33342, resulting in blue fluorescence. Proliferating cells were stained by conjugated reaction of Apollo dye and EdU, resulting in red fluorescence. All images were obtained by a fluorescence microscope (FSX100, Olympus, Japan).

\section{Cell cycle analysis}

Cells were harvested and suspended in $500 \mu$ PBS after transfection for $48 \mathrm{~h}$ and then vortexed gently to obtain a monodispersed cell suspension. Cells were fixed in $70 \%$ ethanol overnight at $4^{\circ} \mathrm{C}$. Ethanol was discarded, and $100 \mu \mathrm{l}$ RNase A was added. The suspension was heated at $37^{\circ} \mathrm{C}$ (water bath). After staining with $\mathrm{Pl}$ and keeping in the dark at room temperature for $30 \mathrm{~min}$, signals were captured from the suspension by flow cytometry (FACS Calibur, USA).

\section{RNA extraction and quantitative RT-PCR}

Total RNA was extracted from the cultured cells using Trizol reagent (Life Technologies, USA) according to the manufacturer's protocol. For miR-183 expression level detection, total RNA was reverse-transcribed with a miR-183-specific RT primer (RiboBio, China) and amplified with PCR primers (RiboBio, China) on the ABI 7300 Real-Time PCR System (Life Technologies, USA). The relative expression of miR-183 was normalized against U6. Real-time RT-PCR for PDCD4 was performed with primers specific for PDCD4 (forward, 5'-TATGATGTGGAGGAGGTGGATGTGA-3'; and reverse, 5'-CCTTTCATCCAAAGGCAAAACTACAC-3') (Frankel et al., 2008), and the relative expression level was normalized against $\beta$-actin (forward, 5'-ATCCGCAAAGACCTGT-3'; and reverse, 5'-GGGTGTAACACTAAG-3'), which was designed by Invitrogen (Life Technologies, USA). The difference between miR-183 and PDCD4 expressions in cells treated with mimic/inhibitor and each NC was calculated using the $2^{-\Delta \Lambda C t}$ method (Livak and Schmittgen, 2001), where $\Delta \mathrm{Ct}=\left(\mathrm{Ct}_{\operatorname{miR}-183 / P D C D 4}-\mathrm{Ct}_{\mathrm{ub} / / \text {-actin }}\right)$ and $\Delta \Delta \mathrm{Ct}=\Delta \mathrm{Ct}_{\text {mimic/ihibitor }}-\Delta \mathrm{Ct}_{\mathrm{NC}}$.

\section{Western blot analysis}

The protein level of PDCD4 was determined by Western blot in EC9706 cells treated with miR-183 mimic. Total proteins (20 $\mu \mathrm{g}$ ) were mixed with $1 \times 6$ loading buffer and boiled for $5 \mathrm{~min}$. $\beta$ actin $(42 \mathrm{KD})$ and PDCD4 (60 KD) were isolated by SDS-PAGE with $10 \%$ separation gel and $5 \%$ spacer gel concentrations. Subsequently, proteins were transferred to polyvinylidene fluoride membrane at $250 \mathrm{~mA}$ for $1.5 \mathrm{~h}$, and the membrane was blocked with $5 \%$ nonfat milk overnight at $4^{\circ} \mathrm{C}$. Primary mouse anti-human $\beta$-actin IgG (Boster, China) and rabbit anti-human PDCD4 IgG (Cell Signaling, USA) were optimized at 1:600 and $1: 1,000$, respectively, and incubated with the membrane at $4^{\circ} \mathrm{C}$ overnight. The membrane was incubated with the secondary antibody (dilution 1:5,000 and 1:3,000, respectively) (Santa Cruz, USA and Cell Signaling, USA) for $1 \mathrm{~h}$ at room temperature after washing with TBST buffer. Signals were visualized with chemiluminescent substrate (Pierce, USA) by exposure to films.

\section{3'UTR luciferase reporter assay}

The wild type and mutant 3'UTR of PDCD4 were cloned into the pmiR-RB miRNA reporter vector (synthesized by Ribobio, China) to confirm direct target association. The wild type contained binding sites of PDCD4 3'UTR with miR-183. The se- 
Table 1. Highly dysregulated genes between cells treated with miR-183mimic and cells with negative control

\begin{tabular}{lccc}
\hline NCBI accession & Fold change & Gene symbol & Description \\
\hline Top 10 up-regulated genes & & & \\
NM_002575 & 4.3486104 & SERPINB2 & Serpin peptidase inhibitor \\
NM_001098612 & 3.8944647 & SIGLEC14 & Sialic acid binding Ig-like lectin 14 \\
NM_145653 & 3.4289598 & TCEB3C & Transcription elongation factor B polypeptide 3C \\
NM_001105569 & 3.062075 & MSGN1 & Mesogenin 1 \\
NM_032165 & 2.694109 & LRRIQ1 & Leucine-rich repeats and IQ motif containing 1 \\
NM_014059 & 2.629876 & C13orf15 & Chromosome 13 open reading frame 15 \\
NR_024356 & 2.500537 & FBLL1 & Fibrillarin-like 1 \\
NM_201266 & 2.4455626 & NRP2 & Neuropilin 2 (NRP2), \\
NM_006708 & 2.4195414 & GLO1 & Glyoxalase I (GLO1) \\
NM_017801 & 2.2300541 & CMTM6 & CKLF-like MARVEL transmembrane domain containing 6 \\
Top 10 down-regulated genes & & & Major histocompatibility complex, class II, DR beta 5 \\
NM_002125 & 4.268639 & HLA-DRB5 & Integrin, beta 1 \\
NM_133376 & 2.810795 & ITGB1 & Presenilin 2 \\
NM_005931 & 2.5367131 & MICB & MHC Class I Chain-Related Protein B \\
NM_000447 & 2.4689913 & PSEN2 & Programmed cell death 4 \\
NM_145341 & 2.4097233 & PDCD4 & CTD small phosphatase 1 \\
NM_021198 & 2.3519185 & CTDSP1 & cDNA, chr7:7918719-7918778 \\
XR_040869 & 2.2505813 & KIAA0101 \\
NM_014736 & 2.2059736 & KIAA0101 & ARPP19 \\
NM_006628 & 2.1861598 & CDKL5 & cAMP-regulated phosphoprotein \\
NM_003159 & 2.1652699 & Cyclin-dependent kinase-like 5
\end{tabular}

quence that was complementary to the binding sites (positions 260-266 of PDCD4 3'UTR:GUGCCAU) was replaced by CACGGTA for mutagenesis (Fig. 6A). The sequence of Renilla luciferase was constructed for reporter fluorescence (Rluc) in these plasmid vectors, whereas the sequence of firefly luciferase was designed for calibration fluorescence (Luc) as an internal reference to monitor the efficiency of plasmid expression. Cells $\left(1 \times 10^{4}\right)$, vectors $(100 \mathrm{ng})$, and miRNA mimic $(1.5 \mathrm{pmol})$ in each well (in a 96-well plate) were mixed in RPMI1640 medium with $10 \%$ FBS and incubated for $48 \mathrm{~h}$. Luciferase activity was measured using a dual-luciferase reporter assay system (Promega, USA) after incubation and was read with Mithras LB 940 (Berthold Technologies, Belgium). Luciferase signal ratio (Rluc/Luc) was calculated for each construct.

Statistical analysis of data

The prediction of miRNA targets was performed by using Target Scan (release 6.2, http://www.targetscan.org/) and miRDB (http://mirdb.org/miRDB/). All results were expressed as means \pm $S D$. Student's $t$-test was performed to determine the differences of miRNA and mRNA expression levels. One-way ANOVA was used for luciferase reporter assay analysis. The relationship between miR-183 and PDCD4 expression was analyzed using the Pearson correlation coefficient. All experiments were performed in triplicate. All statistical analyses were performed on SPSS 18.0. $p<0.05$ was considered statistically significant.

\section{RESULTS}

Profiling of differentially expressed genes in EC9706 cells treated with miR-183 mimic and NC

After transfection for $48 \mathrm{~h}$, miR-183 expression was greatly changed in EC9706, according to the results of real-time RT-
PCR analysis. Compared with cells treated with the NC, the expression of miR-183 in cells with the mimic was up-regulated by $2^{12.75}$-fold $(p<0.001)$, whereas the expression in cells with inhibitor was down-regulated by 1.99 -fold $(p=0.002)$. Microarray profiling revealed 100 up-regulated genes and 83 downregulated genes. The markedly changed genes are shown in Table 1. The remarkably decreased genes in miR-183 mimictreated cells were HLA-DRB5, ITGB1, MICB, PSEN2 and PDCD4. The changes in these genes were 4.2- to 2.4-fold. Most of these genes are critically related to tumorigenesis. Cluster analysis based on the differentially expressed mRNAs was used to successfully separate the miR-183 mimic treated cells from the NC, thereby revealing the consistency in each case. The cumulative distribution of the log2-transformed gene expression fold changes for genes predicted by TargetScan and other genes was plotted (Fig. 1). A significant shift for genes predicted as targets of miR-183 compared to those nontarget genes indicates the miR-183 target genes predicted is more likely to be repressed $(p<0.001$, Kolmogorov-Smirnov test).

\section{Bioinformatic analysis}

The identified genes showing alterations were annotated in the GO analysis for biological function classification and in the pathway analysis for the elucidation of whole chains of events in miR-183 over-expressing cells. Among all the downregulated genes, the $\mathrm{GO}$ analysis for "biological process", "cellular component", and "molecular function" showed significant enrichments of 13,10 , and 10 terms, respectively. The most significantly enriched GO term under the molecular function category was the cyclin-dependent protein kinase regulator activity, thereby suggesting its critical role in cell cycle progression. TGF-beta signaling and p53 signaling pathways were 


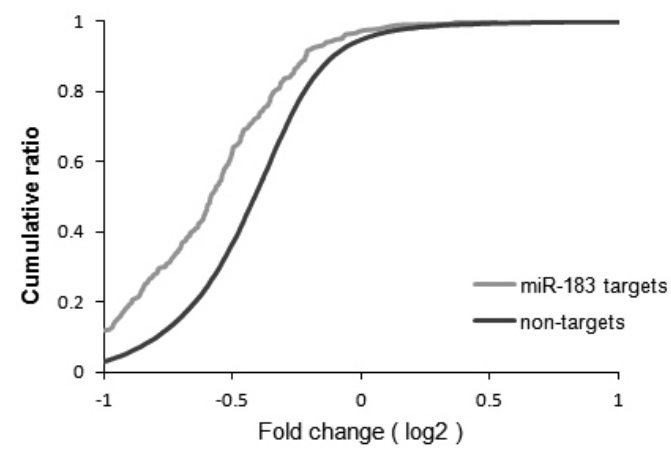

Fig. 1. Cumulative distribution plots of log2-transformed gene expression fold changes for genes containing miR-183 target sites predicted by TargetScan and all other expressed genes of nontargets after transfection with miR-183 mimic and NC in EC9706. The CDFs for targets of TargetScan and non-target genes are significantly different $(p<0.001)$ by the Kolmogorov-Smirnov test.
A miR-183 mimic

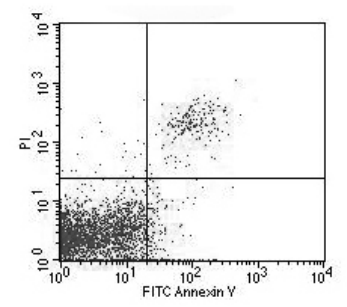

negative control

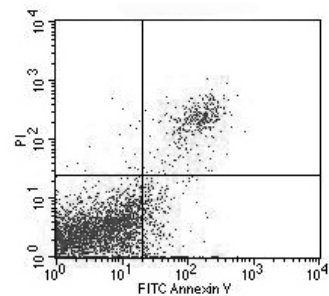

B

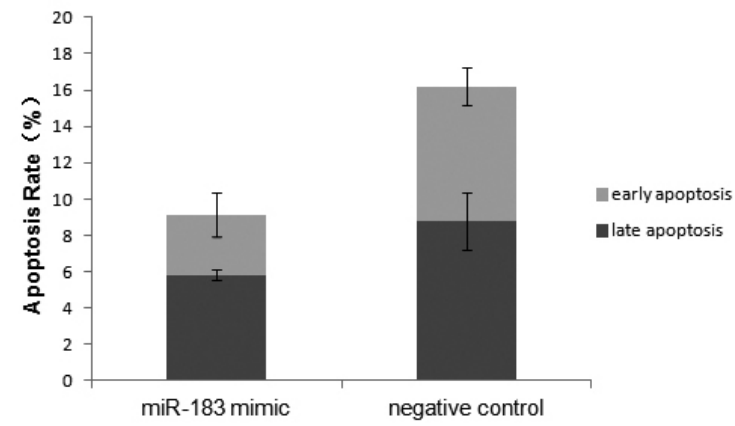

Fig. 2. Up regulation of miR-183 resisted serum deprivationinduced apoptosis. (A) Annexin-V FITC/PI assay was used to detect apoptotic cells in EC9706 cells treated with miR-183 mimic/NC. The lower right quadrant represents the early apoptosis. The upper right quadrant represents the late apoptosis. (B) The rates of apoptosis in EC9706 cells were quantified. The early apoptosis rates and late apoptosis rates in cells transfected with miR-183 mimic were both significantly decreased compared with negative control $(p<0.05)$.

significantly enriched in the KEGG pathway analysis. Both pathways are classic signaling pathways in tumorigenesis. Cell cycle arrest was the major output that resulted from the deregulation of the above pathways. This result suggested that these altered cancer-related genes may play important roles in ESCC Additionally, we noticed the frequent occurrence of genes involved in the regulation of apoptosis, proliferation, differentiation, and migration of the down-regulated genes. The en-
A miR-183 inhibitor

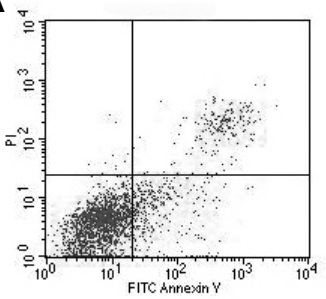

negative control

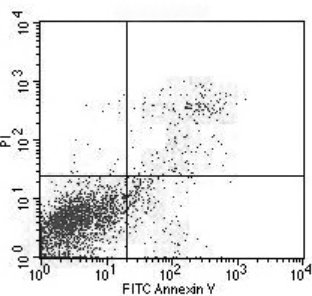

B

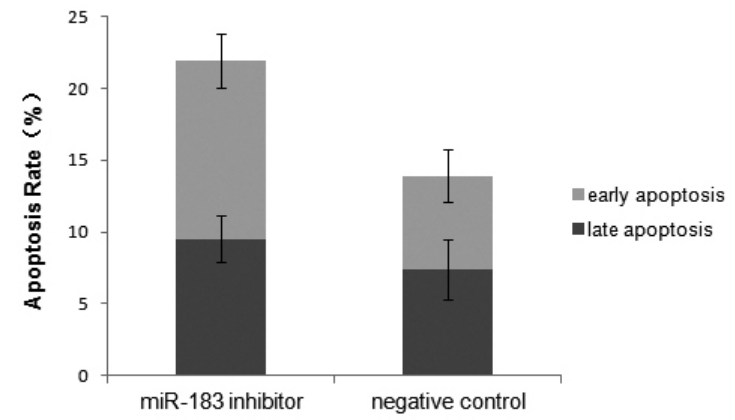

Fig. 3. Down regulation of miR-183 rescued the inhibition of serum deprivation-induced apoptosis. (A) Annexin-V FITC/PI assay was used to detect apoptotic cells in EC9706 cells treated with miR-183 inhibitor/NC as mentioned above. (B) The rates of apoptosis in EC9706 cells were quantified. The early apoptosis rate and late apoptosis rate in cells treated with miR-183 inhibitor were consistently resumed $(p<0.05)$.

riched GO terms for molecular function were also related to protein kinase activity, epithelial cell differentiation, and immune response. KEGG pathway analysis revealed that several classic cancer-related pathways, such as ECM-receptor interaction, focal adhesion, and pathways in cancer, were significantly disturbed.

miR-183 inhibits serum deprivation-induced apoptosis in EC9706 cells

After $48 \mathrm{~h}$ of transfection, serum-containing medium was replaced by serum-free medium for $24 \mathrm{~h}$ to induce apoptosis. Results showed that the early apoptosis rate was reduced in the cells with miR-183 mimic compared with that in the NC (3.31 \pm 1.20 vs. $7.43 \pm 1.01, p<0.001)$, and late apoptosis rate also decreased (5.83 \pm 0.29 vs. $8.77 \pm 1.59, p<0.01)$ (Fig. 2). A rescue experiment was conducted via miR-183 inhibitor to determine the function of $\mathrm{miR}-183$ in apoptosis regulation. The inhibitory effects of miR-183 on early apoptosis rate (12.39 \pm 1.89 vs. $6.56 \pm 1.87, p<0.001)$ and on late apoptosis rate $(9.53$ \pm 1.58 vs. $7.36 \pm 2.42, p<0.05$ ) were rescued, as shown in Fig. 3 . These results indicated that miR-183 can suppress serum deprivation-induced apoptosis in EC9706 cells.

Over-expression of miR-183 promoted cell proliferation and G1/S transition in EC9706 cells

miR-183 functions as an oncogene in ESCC. Thus, we examined whether miR-183 could modulate proliferation in EC9706 cells. EdU incorporation experiments and cell cycle analysis were performed in EC9706 cells transfected with miR-183 mimic and NC. EdU analysis showed that more than half of the cells transfected with the miR-183 mimic were EdU positive. However, 
A

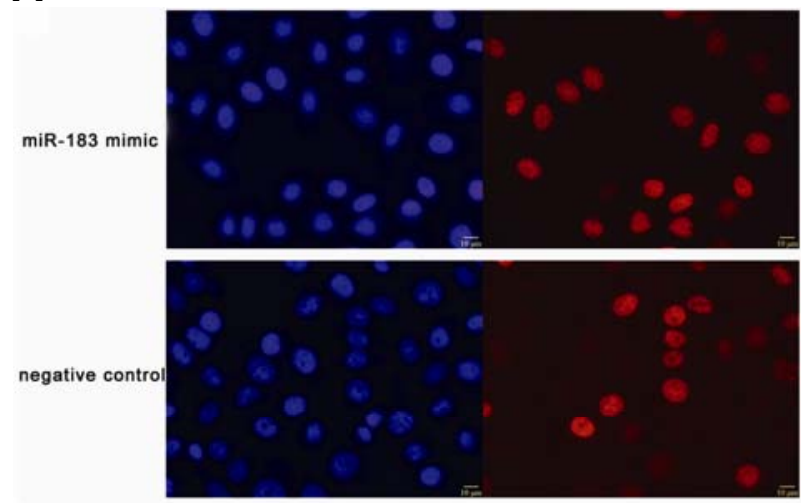

B

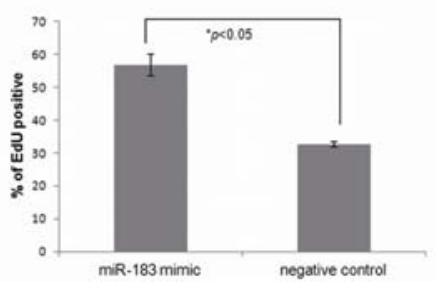

Fig. 4. miR-183 promoted cell proliferation in EC9706 cells by EdU analysis. (A)Proliferating cells were labeled after conjugated reaction of Apollo dye and EdU (red). Cell nuclei stained with Hoechst 33342 (blue) represents a total population of cells. The images are representative of the results obtained. (B) The percentages of proliferating cells were quantified. The figures show a significant increase $(p<0.05)$ in the proliferation rate in EC9706 cells treated with miR-183 mimic.

only one-third of the control cells were EdU positive (Figs. 4A and $4 \mathrm{~B}$ ). The results indicated that the over-expression of miR183 significantly accelerated the cell proliferation rate in EC9706 cells $(p<0.05)$. Moreover, the cell cycle distribution of EC9706 cells showed that the percentage of cells at G1 phase significantly decreased in miR-183 mimic-transfected cells compared with NC $(p<0.05)$ (Figs. 5A and 5B), but the percentages at $S$ and $\mathrm{G} 2$ phases showed no statistical difference. Thus, over-expression of miR-183 accelerated cell cycle progression.

miR-183 regulated the expression of PDCD4 at protein levels We analyzed the putative target genes using bioinformatics approaches, namely, TargetScan (release 6.2, http://www. targetscan.org/) and miRDB (http://mirdb.org/miRDB/), to identify the downstream genes of miR-183. Both approaches predicted miRNA targets with different rules of targeting. Target Scan involves the use of UCSC whole-genome alignments to identify $3^{\prime} U T R$ sequences and predicts biological targets of miRNAs by searching for the presence of conserved 8 mer and 7 mer sites that match the seed region of each miRNA (Thomson et al., 2011). miRDB involves the use of a bioinformatics tool MirTarget2, which is developed by analyzing thousands of genes impacted by miRNAs with an SVM learning machine (http://mirdb.org/miRDB/). We combined the results from the two prediction programs to find bona fide targets. PDCD4, which was discovered by both microarray profiling and bioinformatics predictions, was selected for further validation (Fig. 6A). RT-qPCR showed that PDCD4 slightly decreased in
A
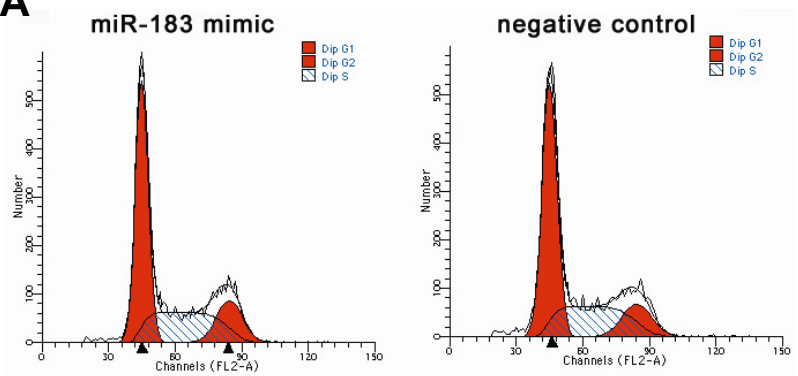

B

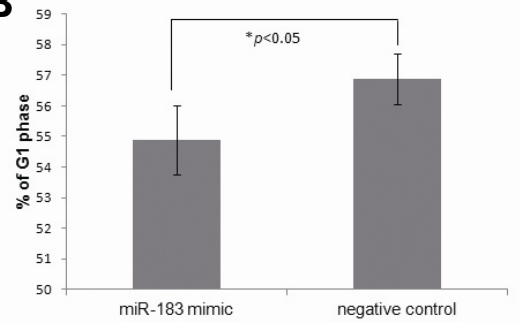

Fig. 5. miR-183 accelerated G1/S transition in EC9706 cells by cell cycle analysis. (A) Flow cytometry analysis distinguish cells in different phases of cell cycle. (B) The cell percentages at different phases showed a cell cycle acceleration in G1/S transition when treated with miR-183 mimic $(p<0.05)$

EC9706 cells treated by miR-183 mimic and increased in miR183 inhibitor-treated cells, but no statistical differences were observed. Degrading target mRNAs did not seem to be the major way by which miR-183 regulated PDCD4. Western blot analysis confirmed that miR-183 significantly reduced the protein level of PDCD4 (fold change $=0.76, p<0.05$ ) (Figs. 6B and $6 \mathrm{C}$ ). These results indicated that miR-183 negatively regulated the expression of PDCD4 mainly by inhibiting translation.

miR-183 suppresses PDCD4 expression by binding to 3'UTR sequences

Luciferase reporter assay was performed to validate the direct target association of miR-183 and PDCD4. Figure 7 shows the luciferase signals from co-transfection of PDCD4 3'UTR constructs (with or without miR-183 binding site mutation), miR-183 mimic, and NC. ANOVA analysis indicated that miR-183 significantly inhibited the expression of wild $3^{\prime} U T R$ constructs of PDCD4 $(p<0.01)$ compared with other treatments. Cotransfection of miR-183 with wild-type plasmid showed a 2.12fold decrease compared with the mutant type with NC. These data suggest that miR-183 may repress the expression of PDCD4 by binding to the specific sequences in the $3^{\prime}$ UTR of PDCD4.

Association of PDCD4 mRNA level with miR-183 in ESCC cell line and esophageal tissues

The relative expression of PDCD4 mRNA and miR-183 was determined in two ESCC cell lines and a human esophageal epithelial cell line (Het-1A) to explore the actual association between PDCD4 and miR-183. EC9706 showed the lower expression level of miR-183 and the higher expression level of PDCD4 against Het-1A (Fig. 8A). Pearson correlation analysis showed the significant correlation of miR-183 and PDCD4 in both cell lines $(\mathrm{R}=-0.81, p<0.01)$.

The correlation in esophageal tissues was also determined. 
A

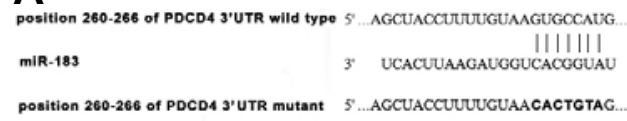

B
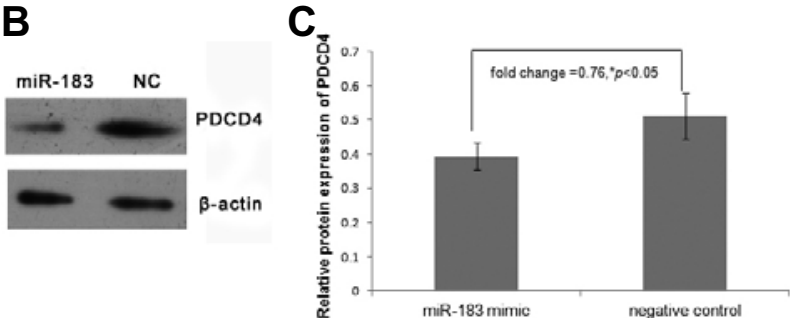

Fig. 6. miR-183 down-regulated PDCD4. (A) The potential miR-183 seed region at the binding site in PDCD4 3'UTR (position 260-266) was computationally predicted by TargetScan. The corresponding mutated nucleotides of the PDCD4 3'UTR was labeled in bold bellow. $(B, C)$ show protein expression of PDCD4 in EC9706 cells treated with miR-183 mimic/NC normalized against $\beta$-actin. The PDCD4 protein expression significantly decreased by miR-183 over-expression (fold change $=0.76, p<0.05$ ).

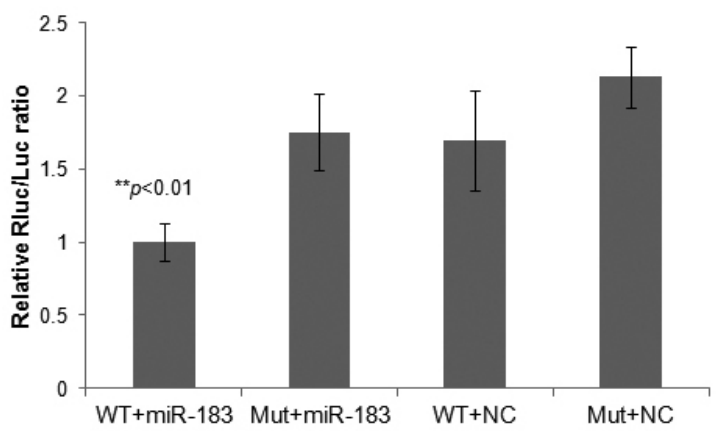

Fig. 7. miR-183 binds to the $3^{\prime} U T R$ of PDCD4 using luciferase reporter assay. Bar represents the relative Rluc/Luc ratio each construct. From left to right are co-transfection of wild type of PDCD4 3'UTR and miR-183 mimic, mutant type of PDCD4 3'UTR and miR183 mimic, wild type and negative control of mimic, mutant type and negative control. miR-183 significantly decreased luciferase activity in $3^{\prime}$ UTR of PDCD4 which suggests that miR-183 directly targets the $3^{\prime} U T R$ of PDCD4. WT, plasmid construct containing wild type of PDCD4 3'UTR. Mut, plasmid construct containing mutant type of PDCD4 3'UTR. NC, negative control for miR-183 mimic. ${ }^{*} p<0.01$.

With the log10-transformed data, the Pearson correlation analysis showed a significant negative correlation between miR-183 and PDCD4 ( $\mathrm{R}=-0.189, p=0.016$ ) (Fig. 8B). However, PDCD4 expression levels in cancer tissues and adjacent non-tumor tissues were not statistically different. The results indicated that miR-183 could affect PDCD4 expression.

\section{DISCUSSION}

miR-183 is a member of the miR-96-182-183 cluster located in $7 q$, which was a preferential site of translocation (Corcoran et al., 1999), loss of heterozygosity (Achille et al., 1996; Bieche et al., 1992), and amplification (Koo et al., 2001). In addition, frequent gains at this site were observed in ESCC (Kwong et al.,
A

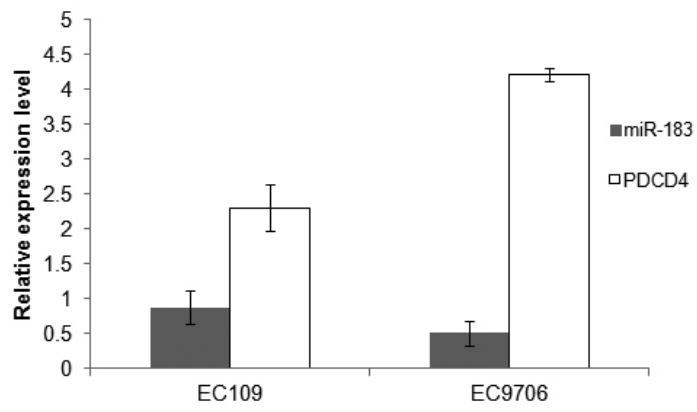

B

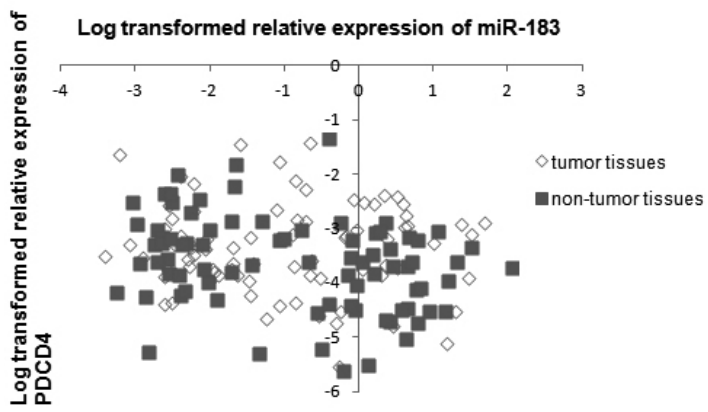

Fig. 8. Association of miR-183 and PDCD4 in ESCC cell lines and patients. (A) shows the relative expression of miR-183 and PDCD4 in EC9706 and EC109 with respect to Het-1A (a human esophageal epithelial cell line). Correlation analysis showed a significant correlation in ESCC cell lines $(R=-0.81, p<0.01)$. (B) shows the scatter plot of miR-183 and PDCD4 expression in ESCC patients. Both the tumor tissues (diamond) and non-tumor tissues (square) showed a significant negative correlation between miR-183 and PDCD4 $(R=-0.189$, $p=0.016)$.

2004), thereby indicating the up-regulation of miR-183 in ESCC. The target genes of miR-183 in malignancy were reported in several cancers. Sarver et al. (2010) demonstrated that miR183 promoted cell migration in multiple tumor types, including synovial sarcoma, rhabdomyosarcoma, and colon cancer, by targeting EGR1. Tanaka et al. (2013) reported that miR-183 was up-regulated in malignant gliomas and mediated by targeting IDH2. miR-183 increased cell growth and motility by targeting Dkk-3 and SMAD4 in prostate cancer (Ueno et al., 2013). However, miR-183 decreased cell invasion and migration capacities by targeting ITGB1 in cervical cancer (Li et al., 2010a) and by targeting Ezrin in osteosarcoma (Zhu et al., 2012). The conflicting roles of miR-183 in different cancers may be caused by tissue dependence. miR-183 would likely target diverse downstream genes through which it would exert different biological functions on cancer cells.

In the present study, we found that the over-expression of miR-183 can suppress serum deprivation-induced apoptosis, and the repression of apoptosis could be rescued when miR183 expression was reduced via an miR-183 inhibitor. In addition, miR-183 accelerated cell progression in G1/S phase transition and promoted cell proliferation. Thus, miR-183 promoted ESCC tumorigenesis at phenotype level from the cytobiological features. A total of 183 differentially expressed genes were screened, including 100 up-regulated genes and 83 downregulated genes between miR-183 mimic treated cells and NC, according to microarray profiling results. These altered genes were critically related to a number of processes or pathways, 
including cell cycle progression, cell apoptosis, proliferation, differentiation, p53 pathway, and TGF- $\beta$ pathway. We speculate that the abovementioned phenotypic changes may be regulated by some downstream genes of miR-183.

miRNA target prediction programs (TargetScan and miRDB) and mRNA microarray profiling were employed to identify the bona fide targets of miR-183. PDCD4 was markedly identified as one of the targets of miR-183. The binding sites of miR-183 were in PDCD4 3'UTR. The PDCD4 gene was originally considered to be up-regulated in apoptotic cells, and this gene encoded a protein that is localized to the nucleus in proliferating cells (Shibahara et al., 1995). Furthermore, results of previous studies confirmed that PDCD4 is an important tumor suppressor gene (Gaur et al., 2011; Wei et al., 2012; Zhang et al., 2010). The present study confirmed that over-expression of miR-183 could inhibit PDCD4 protein expression. Moreover, the expression level of PDCD4 was related to the expression of miR-183, which was confirmed in esophageal cancer cell lines and in a human esophageal epithelial cell line. Furthermore, the correlation analysis showed a significantly negative correlation in tumor tissues and adjacent non-tumor tissues, thereby suggesting that the expression of miR-183 was closely related to PDCD4 expression. In addition, miR-183 might exert oncogenic function by targeting PDCD4 in ESCC. An early report on miR183 in hepatocellular carcinoma by $\mathrm{Li}$ et al. (2010b) demonstrated that miR-183 could target PDCD4 by RT-PCR detection and Western blot. The role of miR-183 in ESCC requires further study given that miR-183 may play conflicting roles in different tumors. The role of miR-183 in esophageal cancer was explored for the first time in the present study. We verified the negative correlation between miR-183 expression and PDCD4 expression in a population study, thereby confirming target association. The parallel results in the two studies confirmed the oncogenic role of miR-183 and PDCD4 as a downstream target in tumors.

PDCD4 was frequently reduced in a variety of tumors (Fassan et al., 2010; Ma et al., 2013; Santhanam et al., 2010; Wang et al., 2010). As a tumor suppressor gene, PDCD4 is recognized as a gene that is up-regulated after the initiation of apoptosis (Shibahara et al., 1995; Zhang et al., 2006). In gastric cancer, PDCD4 can regulate the progress of apoptosis by down-regulating FLIP, which is a negative regulator of apoptosis (Wang et al., 2010). In hepatocellular carcinoma, PDCD4 mediates TGF- $\beta 1$-induced apoptosis by stimulating the synthesis of PDCD4 mRNA via the Smad signaling pathway (Matsuhashi et al., 2014; Zhang et al., 2006). A report from Eto et al. (2012) demonstrated that the loss of PDCD4 increased procaspase-3 expression, thereby leading to its activation and PARP cleavage even without apoptotic stimuli. Moreover, the cells were sensitized to apoptosis. These findings indicate that PDCD4 participates in tumorigenesis by regulating apoptosis. Tumorigenesis is associated with increased cell proliferation and apoptosis reduction. PDCD4 can induce cell cycle arrest at G1 stage in ovarian cancer (Lankat-Buttgereit and Goke, 2009; Wei et al., 2012), and this result is consistent with our findings in the present study. As for molecular mechanisms, PDCD4 induced the expression of p27 and p21 (Wei et al., 2012), which are critically related to cell cycle progression and proliferation. In addition, PDCD4 knockdown can up-regulate cyclinD1expression through NF-kB activation and GSK3 $\beta$ phosphorylation (Guo et al., 2011). The abovementioned findings illustrate that PDCD4 loss results in cell cycle acceleration. PDCD4 also exerts inhibitory effects on cancer cell migration and invasion (Jiang et al., 2010; Zhang et al., 2009). PDCD4 expression is associated with nodal metastasis and invasion; thus, PDCD4 is a clinically relevant biomarker with prognostic value in oral carcinoma (Reis et al., 2010). Moreover, PDCD4 down-regulation is a reliable biomarker of early-stage squamous cell esophageal cancer (Fassan et al., 2012). Consequently, we were able to speculate that the down-regulation of PDCD4 is a frequent event in tumorigenesis and exerts tumorsuppressive function in tumor cell movement, invasion, proliferation, and apoptosis.

We found that miR-183 facilitates the progression of ESCC by down-regulating PDCD4 expression. Our results can be the basis for further analysis of miR-183 and PDCD4 in vivo to develop a new potential diagnostic and therapeutic target for ESCC screening and treatment.

\section{ACKNOWLEDGMENTS}

This study is supported by the National Natural Science Foundation of China (No. 81172747, 81072259, 30800891, 81111140396) Research Fund for the Doctoral Program of Higher Education of China (No. 200802861045), Natural Science Foundation of Jiangsu province, China (No. BK2010407)

\section{REFERENCES}

Achille, A., Biasi, M.O., Zamboni, G., Bogina, G., Magalini, A.R., Pederzoli, P., Perucho, M., and Scarpa, A. (1996). Chromosome $7 q$ allelic losses in pancreatic carcinoma. Cancer Res. 56, 38083813.

Bieche, I., Champeme, M.H., Matifas, F., Hacene, K., Callahan, R., and Lidereau, R. (1992). Loss of heterozygosity on chromosome $7 q$ and aggressive primary breast cancer. Lancet 339, 139-143.

Borel, C., and Antonarakis, S.E. (2008). Functional genetic variation of human miRNAs and phenotypic consequences. Mamm Genome 19, 503-509.

Calin, G.A., and Croce, C.M. (2006). MicroRNA signatures in human cancers. Nat. Rev. Cancer 6, 857-866.

Corcoran, M.M., Mould, S.J., Orchard, J.A., Ibbotson, R.E., Chapman, R.M., Boright, A.P., Platt, C., Tsui, L.C., Scherer, S.W., and Oscier, D.G. (1999). Dysregulation of cyclin dependent kinase 6 expression in splenic marginal zone lymphoma through chromosome $7 \mathrm{q}$ translocations. Oncogene 18, 6271-6277.

Dubecz, A., Gall, I., Solymosi, N., Schweigert, M., Peters, J.H. Feith, M., and Stein, H.J. (2012). Temporal trends in long-term survival and cure rates in esophageal cancer: a seer database analysis. J. Thorac. Oncol. 7, 443-447.

Eto, K., Goto, S., Nakashima, W., Ura, Y., and Abe, S.I. (2012). Loss of programmed cell death 4 induces apoptosis by promoting the translation of procaspase-3 mRNA. Cell Death Differ. 19, 573-581.

Fang, L., Du, W.W., Yang, W.N., Rutnam, Z.J., Peng, C., Li, H.R., O'Malley, Y.Q., Askeland, R.W., Sugg, S., Liu, M.Y., et al. (2012) MiR-93 enhances angiogenesis and metastasis by targeting LATS2. Cell Cycle 11, 4352-4365.

Fassan, M., Cagol, M., Pennelli, G., Rizzetto, C., Giacomelli, L., Battaglia, G., Zaninotto, G., Ancona, E., Ruol, A., and Rugge, M. (2010). Programmed cell death 4 protein in esophageal cancer. Oncol. Rep. 24, 135-139.

Fassan, M., Realdon, S., Pizzi, M., Balistreri, M., Battaglia, G. Zaninotto, G., Ancona, E., and Rugge, M. (2012). Programmed cell death 4 nuclear loss and miR-21 or activated Akt overexpression in esophageal squamous cell carcinogenesis. Dis. Esophagus 25, 263-268.

Frankel, L.B., Christoffersen, N.R., Jacobsen, A., Lindow, M., Krogh, A., and Lund, A.H. (2008). Programmed cell death 4 (PDCD4) is an important functional target of the microRNA miR-21 in breast cancer cells. J. Biol. Chem. 283, 1026-1033.

Gaur, A.B., Holbeck, S.L., Colburn, N.H., and Israel, M.A. (2011). Downregulation of Pdcd4 by mir-21 facilitates glioblastoma proliferation in vivo. Neuro Oncol. 13, 580-590.

Guo, X., Li, W., Wang, Q., and Yang, H.S. (2011). AKT activation by Pdcd4 knockdown up-regulates cyclin D1 expression and promotes cell proliferation. Genes Cancer 2, 818-828. 
Guo, P., Huang, Z.L., Yu, P., and Li, K. (2012). Trends in cancer mortality in China: an update. Ann. Oncol. 23, 2755-2762.

Han, Y., Chen, J., Zhao, X., Liang, C., Wang, Y., Sun, L., Jiang, Z., Zhang, Z., Yang, R., Li, Z., et al. (2011). MicroRNA expression signatures of bladder cancer revealed by deep sequencing. PLoS One 6, e18286.

He, J., and Chen, W. (2012). Chinese Cancer Registry Annual Report (Beijing, China: Military Medical Sciences Press).

$\mathrm{Hu}$, Y., Correa, A.M., Hoque, A., Guan, B., Ye, F., Huang, J., Swisher, S.G., Wu, T.T., Ajani, J.A., and Xu, X.C. (2011) Prognostic significance of differentially expressed miRNAs in esophageal cancer. Int. J. Cancer 128, 132-143.

Hvid-Jensen, F., Pedersen, L., Drewes, A.M., Sorensen, H.T., and Funch-Jensen, P. (2011). Incidence of adenocarcinoma among patients with Barrett's esophagus. N. Engl. J. Med. 365, 13751383.

Jiang, Y., Zhang, S.H., Han, G.Q., and Qin, C.Y. (2010). Interaction of Pdcd4 with elF4E inhibits the metastatic potential of hepatocellular carcinoma. Biomed. Pharmacother. 64, 424-429.

Koo, S.H., Ihm, C.H., Kwon, K.C., Park, J.W., Kim, J.M., and Kong, G. (2001). Genetic alterations in hepatocellular carcinoma and intrahepatic cholangiocarcinoma. Cancer Genet. Cytogenet. 130 22-28.

Kwong, D., Lam, A., Guan, X., Law, S., Tai, A., Wong, J., and Sham, J. (2004). Chromosomal aberrations in esophageal squamous cell carcinoma among Chinese: Gain of $12 p$ predicts poor prognosis after surgery. Hum. Pathol. 35, 309-316.

Lankat-Buttgereit, B., and Goke, R. (2009). The tumour suppressor Pdcd4: recent advances in the elucidation of function and regulation. Biol. Cell 101, 309-317.

Li, G.R., Luna, C., Qiu, J.M., Epstein, D.L., and Gonzalez, P. (2010a). Targeting of integrin $\beta 1$ and kinesin $2 \alpha$ by microRNA 183. J. Biol. Chem. 285, 5461-5471

Li, J., Fu, H., Xu, C., Tie, Y., Xing, R., Zhu, J., Qin, Y., Sun, Z., and Zheng, X. (2010b). miR-183 inhibits TGF-beta1-induced apoptosis by downregulation of PDCD4 expression in human hepatocellular carcinoma cells. BMC Cancer 10, 354.

Lin, Y., Totsuka, Y., He, Y., Kikuchi, S., Qiao, Y., Ueda, J., Wei, W., Inoue, M., and Tanaka, H. (2013). Epidemiology of esophageal cancer in Japan and China. J. Epidemiol. 23, 233-242.

Livak, K.J., and Schmittgen, T.D. (2001). Analysis of relative gene expression data using real-time quantitative PCR and the $2^{-\Delta \Delta C}$ method. Methods 25, 402-408.

Ma, G., Zhang, H., Dong, M., Zheng, X.Y., Ozaki, I., Matsuhashi, S., and Guo, K.J. (2013). Downregulation of programmed cell death 4 (PDCD4) in tumorigenesis and progression of human digestive tract cancers. Tumor Biol. 34, 3879-3885.

Matsuhashi, S., Hamajima, H., Xia, J.H., Zhang, H., Mizuta, T. Anzai, K., and Ozaki, I. (2014). Control of a tumor suppressor PDCD4: Degradation mechanisms of the protein in hepatocellular carcinoma cells. Cell. Signal. 26, 603-610.

Patil, V.S., Zhou, R., and Rana, T.M. (2014). Gene regulation by non-coding RNAs. Crit. Rev. Biochem. Mol. Biol. 49, 16-32

Reis, P.P., Tomenson, M., Cervigne, N.K., Machado, J., Jurisica, I., Pintilie, M., Sukhai, M.A., Perez-Ordonez, B., Grenman, R., Gilbert, R.W., et al. (2010). Programmed cell death 4 loss increases tumor cell invasion and is regulated by miR-21 in oral squamous cell carcinoma. Mol. Cancer 9, 238.

Salic, A., and Mitchison, T.J. (2008). A chemical method for fast and sensitive detection of DNA synthesis in vivo. Proc. Natl.
Acad. Sci. USA 105, 2415-2420.

Santhanam, A.N., Baker, A.R., Hegamyer, G., Kirschmann, D.A and Colburn, N.H. (2010). Pdcd4 repression of lysyl oxidase inhibits hypoxia-induced breast cancer cell invasion. Oncogene 29, 3921-3932.

Sarver, A.L., Li, L.H., and Subramanian, S. (2010). MicroRNA miR183 functions as an oncogene by targeting the transcription factor EGR1 and promoting tumor cell migration. Cancer Res. 70, 9570-9580.

Shibahara, K., Asano, M., Ishida, Y., Aoki, T., Koike, T., and Honjo, T. (1995). Isolation of a novel mouse gene MA-3 that is induced upon programmed cell death. Gene 166, 297-301.

Tanaka, H., Sasayama, T., Tanaka, K., Nakamizo, S., Nishihara, M., Mizukawa, K., Kohta, M., Koyama, J., Miyake, S., Taniguchi, M., et al. (2013). MicroRNA-183 upregulates HIF-1alpha by targeting isocitrate dehydrogenase 2 (IDH2) in glioma cells. $J$. Neuro-Oncol. 111, 273-283.

Thomson, D.W., Bracken, C.P., and Goodall, G.J. (2011). Experimental strategies for microRNA target identification Nucleic Acids Res. 39, 6845-6853.

Ueno, K., Hirata, H., Shahryari, V., Deng, G., Tanaka, Y., Tabatabai, Z.L., Hinoda, Y., and Dahiya, R. (2013). microRNA-183 is an oncogene targeting Dkk-3 and SMAD4 in prostate cancer. Br. J. Cancer 108, 1659-1667.

Wang, W., Zhao, J., Wang, H., Sun, Y., Peng, Z., Zhou, G., Fan, L., Wang, X., Yang, S., Wang, R., et al. (2010). Programmed cell death 4 (PDCD4) mediates the sensitivity of gastric cancer cells to TRAIL-induced apoptosis by down-regulation of FLIP expression. Exp. Cell Res. 316, 2456-2464.

Wei, N., Liu, S.S., Chan, K.K.L., and Ngan, H.Y.S. (2012). Tumour suppressive function and modulation of programmed Cell Death 4 (PDCD4) in ovarian cancer. PLoS One 7, e30311.

Yang, M., Liu, R., Sheng, J.Y., Liao, J., Wang, Y., Pan, E.C., Guo, W., Pu, Y.P., and Yin, L.H. (2013). Differential expression profiles of microRNAs as potential biomarkers for the early diagnosis of esophageal squamous cell carcinoma. Oncol. Rep. 29, 169-176.

Zhang, H., OzakiZ, I., Mizuta, T., Hamajima, H., Yasutake, T., Eguchi, Y., Ideguchi, H., Yamamoto, K., and Matsuhashi, S. (2006). Involvement of programmed cell death 4 in transforming growth factor-beta 1-induced apoptosis in human hepatocellular carcinoma. Oncogene 25, 6101-6112.

Zhang, S.H., Li, J.F., Jiang, Y., Xu, Y.J., and Qin, C.Y. (2009) Programmed cell death 4 (PDCD4) suppresses metastastic potential of human hepatocellular carcinoma cells. J. Exp. Clin. Cancer Res. 28, 71

Zhang, X., Wang, X.Y., Song, X.G., Liu, C.M., Shi, Y.Y., Wang, Y.K., Afonja, O., Ma, C.H., Chen, Y.H.H., and Zhang, L.N. (2010) Programmed cell death 4 enhances chemosensitivity of ovarian cancer cells by activating death receptor pathway in vitro and in vivo. Cancer Sci. 101, 2163-2170.

Zhao, H.E., Guo, M.J., Zhao, G.Y., Ma, Q., Ma, B.A., Qiu, X.C., and Fan, Q.Y. (2012). miR-183 inhibits the metastasis of osteosarcoma via downregulation of the expression of Ezrin in F5M2 cells. Int. J. Mol. Med. 30, 1013-1020.

Zhu, J.F., Feng, Y.P., Ke, Z.F., Yang, Z., Zhou, J.Y., Huang, X.R. and Wang, L.T. (2012). Down-regulation of miR-183 promotes migration and invasion of osteosarcoma by targeting Ezrin. Am J. Pathol. 180, 2440-2451. 